\title{
The role of antinuclear antibody (ANA) profile in diagnosis of systemic lupus erythematosus
}

\section{Introduction}

Systemic Lupus Erythematosus (SLE) is a chronic multi-organ systems autoimmune disease, characterized by the production of predominantly non-organ specific auto antibodies directed to several self molecules found in the nucleous, cytoplasm and cell surface and a wide range of clinical manifestations. ${ }^{1,2}$ This disease typically affects females at far greater rates than males. During reproductive years, the ratio of females to males is nine to one with a lower ratio seen before puberty and a decline later in life. ${ }^{2}$ Causes of premature death associated with SLE are mainly active disease, organ failure (e.g., kidneys), infection, or cardiovascular disease from accelerated atherosclerosis. ${ }^{3}$ SLE may be difficult to diagnosed because its signs and symptoms are not specific and can look like of other disease. ${ }^{4}$ The most widely used classification criteria for SLE are those developed by The American College of Rheumatology published in 1982 and revised in 1997. Diagnosis of SLE is made if a patient satisfied 4 of 11 the criteria. ${ }^{5}$ The criteria are malar rash, discoid rash, photosensitivity, oral ulcers, nonerosive arthritis, pleuritis or pericarditis, renal disorder, neurologic disorder, hematologic disorder, immunologic disorder, positive antinuclear antibody. ${ }^{6}$

\section{Discussion}

This autoimmune disease characterized by the aberrant production of a broad and heterogenous group of autoantibodies. Autoantibodies directed to nuclear (ANAs), cytoplasmic, and cellular membrane antigens are considered the serological hallmark in SLE. ANAs consist of various types of autoantibodies characterized by different antigen specificities. These nuclear antigens include single strand (ss) and double strand (ds) DNA (deoxyribonucleic acid), histone proteins, nucleosome (histone-DNA complex), centromere proteins, and extractable nuclear antigens (ENA) (Smith antigen (Sm), Ro, La, ribonucleoprotein), etc. Common autoantibody - mediated mechanisms of damage in SLE include immune complex mediate damage, cell surface binding and cytotoxicity, reactivity with autoantigens expressed on apoptotic or activated cell surface, penetration into living cells, and binding to cross reactive extracellular molecules. Even though the presence of autoantibodies in SLE has been known, for more than 60 years, still nowadays a great effort is being made to understand the pathogenetic, diagnostic, and prognostic meaning of such Autoantibodies. ${ }^{7,8}$

The traditional methods for detecting ANA are indirect immunofluorescence (IIF) and enzyme immunoassay (ELISA). ${ }^{9}$ In general, if a patient presents clinical manifestations of an autoimmune disease, the first test to be requested is ANA detection using indirect immunofluorescence using HEp-2cells, due to its great sensitivity. The different possible patterns, the intensity, and the titers obtained by consecutive dilutions must be carefully examined, The usefulness of this testing has been evaluated in retro- spective studies of patients with systemic rheumatic disease (SRD), and it has been proven that its positive predictive value is low due to the relatively large amount of false positive results. ${ }^{10}$ ANA pattern is reffered to the pattern of
Volume 2 Issue 5 - 2017

\author{
Chelvi Wijaya, Uleng Bahrun \\ Department of Clinical Pathology, Hasanuddin University, \\ Indonesia
}

Correspondence: Chelvi Wijaya, Department of Clinical Pathology, Hasanuddin University, Makassar, Indonesia, Tel +628I 1467670, Email chelviw@gmail.com

Received: September 04, 2017 | Published: October 13, 2017

nuclear fluorescence observed under fluorescence microscopy. Certain patterns of fluorescence are associated with certain nuclear antigens and specific disease. Patterns of staining provide a clue to the category of nuclear antigen involved and are dependent upon the type of substrate used, and to a certain extent, the experience of the technician. Reliance on ANA patterns has largely been replaced by identification of specific antinuclear antibodies through the ANA profile. ${ }^{11}$

ANA profile with immunoblotting or ELISA methods can detect some antibodies such as seen in Table $1 .{ }^{9,12,13}$ Double stranded DNA antibodies (anti-dsDNA) have been considered useful and valuable in the diagnosis of SLE. ${ }^{1}$ Anti-Sm antibodies are a highly specific marker for SLE and Anti-Sm reactivity is not described in other diseases, however the sensitivity is low. ${ }^{7}$ Anti-dsDNA and anti-Sm antibodies are considered one of the ACR classification criteria along with ANA. ${ }^{1}$ Anti-U1 small nuclear (sn) RNP antibodies are considered pathognomonic for Sharp's syndrome (mixed connective tissue disease or MCTD), but they can be found in $20-30 \%$ of patients with SLE as well. Autoantibodies directed against Ro/SSA and La/ SSB autoantigens were originally identified in patients with Sjögren's syndrome and systemic lupus erythematosus (SLE). Subsequent studies showed that anti-Ro/SSA antibodies may be present in patients with other autoimmune diseases, including systemic sclerosis, idiopathic inflammatory myopathies (IIM), primary biliary cholangitis, and rheumatoid arthritis (RA). ${ }^{12}$

Nucleosomes are considered a major autoantigen in SLE in which they are positive in about $85 \%$ of patients and probably play an important pathogenetic role. Anti-histone antibodies characterize drug-induced lupus. The autoimmune response to ribosomal components is quite specific for SLE. Anti-ribosomal P antibodies occur in $13-20 \%$ of Caucasian SLE patients and in more than $40 \%$ of Asian patients. ${ }^{7}$ Furthermore, there is a correlation between the presence of some autoantibodies and clinical manifestations. AntidsDNA antibodies contribute to the end-stage lupus nephritis. ${ }^{7}$ Antinucleosome antibodies have diagnostic value and play role in the assessment of disease activity especially active renal disease and may predict disease outcome. ${ }^{13}$ 
Table I Autoantibodies detected in ANA Profile

\begin{tabular}{ll}
\hline \multicolumn{2}{l}{ Autoantibodies } \\
\hline nRNP/Sm, & CENP B, \\
Sm, & PCNA, \\
SS-A, & dsDNA, \\
Ro-52, & nucleosomes, \\
SS-B, & histones, \\
Scl-70, & ribosomal P-proteins, \\
PM-Scl, & AMA M2 \\
Jo-I, & \\
\hline
\end{tabular}

\section{Conclusion}

Antinuclear Antibody profile has some advantages over ANA test in diagnosis of SLE due to its capability of detecting specific autoantibodies. Some autoantibodies are found to be correlated with disease activity and organ involvement.

\section{Acknowledgements}

None.

\section{Conflict of interest}

The author declares no conflict of interest.

\section{References}

1. Bertolaccini M, Hughes G, Khamastha M. Systemic lupus erythematosus. In: Shoenfeld Y, et al. editors. Diagnostic Criteria in Autoimmune Disease. USA: Humana Press; 2008. p. 3-7.

2. Morris J, Putterman C. Gender differences in the pathogenesis and outcome of lupus and of lupus nephritis. Clinical and Developmental Immunology. 2012. 9 p.
3. CDC. Lupus Detailed Fact Sheet; 2017.

4. Dall Era M. Systemic lupus erythematosus. In: Imboden JB, et al. editors. Current Rheumatology Diagnosis and Treatment. 3rd ed. USA: Springer; 2103. p. 18-22.

5. Petri M, Orbai AM, Alarcon GS, et al. Derivation and Validation of Systemic Lupus International Collaborating Clinics Classification Criteria for Systemic Lupus Erythematosus. Arthritis Rheum. 2012;64(8):26772686.

6. ACR. 1997 update of the 1982 American college of rheumatology revised criteria for classification of systemic lupus erythematosus; 2017.

7. Cozzani E, Drosera M, Gasparini G, et al. Serology of Lupus Erythematosus : Correlation Between Immunopathological Features and Clinical Aspects. Autoimmune Disease. 2014. 13 p.

8. Desouky S, Gazzar I, Rashed L, et al. Correlation Between Various Clinical Parameters of Systemic Lupus Erythematosus and Levels of Anti-Histone and Anti- Chromatin Antiboidies. The Egyptian Rheumatologist. 2015;37(3):97-104.

9. Sohn KY, Khan W. ANA Testing from Microscopy to Multiplexing; 2014.

10. Soto M, Becerril M, Chiney A, et al. Predictive value of antinuclear antibodies in autoimmune diseases classified by clinical criteria: Analytical study in a specialized health institute, one year follow-up. Results Immunol. 2015;5:13-22.

11. Hobbs K. Laboratory Evaluation. In: West S editor. Rheumatology Secrets. USA: Elsevier Mosby; 2015. p. 48-53.

12. Donald Bloch. The Anti-Ro/SSA and Anti-La/SSB Antigen-Antibody Systems; 2015.

13. Abdel G, Mansour A, Abdel A, et al. Role of Anti-nucleosome in the diagnosis of systemic lupus erythematosus and as marker for lupus nephropathy. Egypt J Immunol. 2014;21(1):57-65. 